\title{
Role of Lipids and Apolipoproteins in Predicting the Prognosis of Hepatocellular Carcinoma After Resection
}

\author{
Xiao-Chun $\mathrm{Ni}^{1}$ \\ Yong $\mathrm{Yi} \mathbb{D}^{2}$ \\ Yi-Peng $\mathrm{Fu}^{3}$ \\ Xiao-Yan $\mathrm{Cai}^{4}$ \\ Gao Liu ${ }^{2}$ \\ Jin-Long Huang ${ }^{2}$ \\ Wei Gan ${ }^{2}$ \\ Jie $X u^{5}$
}

Shuang-Jian Qiu ${ }^{6}$

'Shanghai Ninth People's Hospital, General Surgery, Shanghai, People's Republic of China; ${ }^{2}$ Liver Cancer Institute, Hepatic Surgery, Shanghai, People's Republic of China; ${ }^{3}$ Obstetrics \& Gynecology Hospital of Fudan University, Shanghai, People's Republic of China; ${ }^{4}$ Shanghai Pudong Gongli Hospital, General Surgery, Shanghai, People's Republic of China; ${ }^{5}$ Shanghai Ninth People's Hospital, Infection Disease, Shanghai, People's Republic of China; ${ }^{6}$ Liver Cancer Institute, Zhongshan Hospital, Fudan University, Shanghai 200032, People's Republic of China

Correspondence: Jie Xu

Shanghai Ninth People's Hospital, Infection Disease, 280 Mohe Road, Shanghai, People's Republic of China Email dr.xu@aliyun.com

Shuang-Jian Qiu

Liver Cancer Institute, Zhongshan

Hospital, Fudan University, I80 Fenglin

Road, Shanghai 200032, People's Republic of China

Tel/Fax +86-2I-64037/8I

Email qiushuangjianzs@।63.com
This article was published in the following Dove Press journal: OncoTargets and Therapy

Purpose: To further clarify the association between abnormal levels of serum lipid components as the main features of dyslipidaemia and hepatocellular carcinoma, which remains unclear.

Patients and Methods: We examined the serum level of lipids and apolipoproteins pattern in 471 patients undergoing curative resection for HCC, 193 patients with chronic liver disease, and 104 patients with benign liver diseases. We performed uni- and multivariate analyses to evaluate the predictive roles of lipids and apolipoproteins for recurrence and survival of HCC in a training cohort of 242 patients and then validated in a cohort of 229 patients.

Results: The majority circulating lipid and apolipoprotein levels such as ApoA1, HDL, and LDL in chronic liver disease and HCC were slightly significantly decreased as compared to those in benign lesion. But no significant differential expression patterns of lipids and apolipoproteins were observed between chronic liver hepatitis and HCC. Multivariable analysis identified ApoA1 as a key parameter related to recurrence and survival in both training and validation cohorts. Moreover, we further demonstrated that low ApoA1 was an independent prognostic factor of poor early recurrence in two cohorts.

Conclusion: Although the alterations of circulating lipids and apolipoproteins were observed in HCC, none of lipids or apolipoproteins could serve as a diagnostic marker Serum ApoA1 merits consideration as a novel prognostic marker for patients with HCC undergoing surgery since it predicts early recurrence and survival, especially for early stage patients and may improve the prognostic stratification of patients for clinical management and promote HCC clinic outcomes.

Keywords: apolipoprotein, prognosis, hepatocellular carcinoma, surgery

\section{Introduction}

Hepatocellular carcinoma (HCC) is the most prevalent cancer with a dismal prognosis worldwide. ${ }^{1}$ Until now, surgical resection has remained the primary treatment aiming at cure, however the prognosis of patients with HCC remains poor, mainly due to the fact that most patients are diagnosed at advanced stages and a high incidence of tumor recurrence post-surgery. ${ }^{2}$ Despite HCC patients who receive curative therapy being in very early/early stage tumor, early recurrence is considered as an important adverse prognostic factor after curative therapy for HCC.,4 Therefore, sensitive and effective biomarkers are urgently needed for early diagnosis and accurate prediction of prognosis for patients undergoing curative 
resection. Identification of patients after curative surgery who are at high risk of early recurrence allows clinicians to provide active surveillance to detect recurrent $\mathrm{HCC}$ at its earliest stage, and to improve the prognosis of this population when curative therapy may still be feasible.

Many epidemiological studies have consistently linked dyslipidaemia with increased incidence and death from diverse types of cancer. ${ }^{5-7}$ The liver plays a key role in the metabolism of lipids and lipoproteins. The majority of patients with HCC are frequently accompanied by liver cirrhosis, with chronic hepatitis $\mathrm{B}$ and $\mathrm{C}$ as the major etiological agents. ${ }^{8}$ Therefore liver function is clearly impaired in HCC, leading to a distinctly abnormal pattern of serum lipids and lipoproteins. It has been indicated that high-density lipoprotein (HDL) and its major apolipoproteins, ApoA1 and ApoA2, are frequently reduced in patients suffering from HCC. ${ }^{9,10}$ It also demonstrates that plasma triglycerides (TG) and total cholesterol (TC) decrease in patients with HCC. ${ }^{11}$ In contrast, Alsabti reported that serum TG and TC in HCC patients were increased when compared to those with cirrhosis. ${ }^{12}$ Ooi et al reported that plasma TG levels in HCC patients were not significantly different compared with controls. ${ }^{13}$ A potential explanation for these conflicting results could be the focus on the level of lipids or lipoproteins, without an extensive list of potential confounders that were not always controlled such as body mass index (BMI) and glucose metabolism agents.

Ahaneku et al analyzed lipid profiles in cirrhosis patients with or without HCC and found that plasma HDL-C, HDL-PL and HDL-C/HDL-PL were significantly lower in HCC patients than those in the controls. ${ }^{14}$ It is suggested that variations in the level of plasma lipids and apolipoproteins may assist in describing the nature of these two forms of liver disease. More striking is the discovery that ApoA1 was differentially expressed between chronic hepatitis $\mathrm{C}$ virus (HCV) infection and HCC patients using Mass Spectrometric analysis, suggested ApoA1 might be a candidate biomarker for early diagnosis, prognosis, and monitoring of HCC. ${ }^{15}$ A similar result was found by Steel et al that ApoA1 had a diagnostic role in patients with HCC compared with hepatitis B virus-positive individuals. ${ }^{16}$ These results suggested that plasma lipid profile might be a potential candidate to differentiate HCC from other liver disease and normal populations, and acted as diagnostic biomarkers of HCC. However, the limitations of the studies mentioned above are obvious, such as small numbers of cases enrolled and the techniques used, unsuitable for large-scale analysis in clinical application. Therefore, the accuracy and reliability of the experimental results mentioned have yet to be further verified.

So far sparse studies have investigated the use of serum lipids as biomarkers for diagnosing $\mathrm{HCC}$ and predicting the recurrence or survival of patients with HCC. In this study, we investigated the blood lipids and apolipoproteins pattern including ApoA1, ApoB, ApoE, TC, TG, HDL, and low-density lipoprotein (LDL) in HCC, and other liver diseases including chronic hepatitis, cirrhosis and benign disease, and explored to identify their plausibility as novel diagnostic biomarker candidates for HCC. Furthermore, we analyzed the associations of circulating lipids and apolipoproteins levels with clinicopathological characteristics and prognosis of the patients with HCC.

\section{Patients and Methods}

\section{Study Population}

A totalof 471 patients with primary $\mathrm{HCC}$ who underwent curative liver resection between December 2010 and January 2012 at Liver Cancer Institute, Zhongshan Hospital, Fudan University were included in this retrospective study. All patients had histologically confirmed HCC. None of the patients had received presurgical cancer treatment or were suffering from a recurrence of HCC or from any other known malignancy. The patients were randomly divided into a training cohort $(\mathrm{n}=242)$ and a validation cohort $(\mathrm{n}=229)$. In addition, 140 patients with chronic hepatitis and 53 with cirrhosis, and 104 benign liver diseases, including hepatic hemangioma, hepatic cyst and focal nodular hyperplasia were also enrolled in this study. Benign patients had normal liver function in this cohort relative to normal, age matched donors. The present study was approved by the Research Ethics Committee of Zhongshan Hospital. This study also complied with the Declaration of Helsinki, and written informed consent was obtained from all patients including the parents or legal guardians of patients under the age of 18 .

All lipid and apolipoprotein blood samples were collected at baseline from surgery participants before operation and non-surgery participants during first week of admission. Lipids and apolipoproteins profiles were studied descriptively in relation to potential confounders of metabolism including BMI, blood concentrations of $\mathrm{C}$ reactive protein (CRP) as a biomarker for systemic 
inflammation, fasting blood glucose, Hemoglobin $\mathrm{A} 1 \mathrm{C}$ as well as insulin resistance (C peptide, insulin). As an index of insulin resistance, we used the homeostatic model assessment of insulin resistance (HOMA2-IR). The cutoff values for each variable were based on the range of clinical normals. Clinical stages of tumor were determined according to the TNM classification system of International Union Against Cancer. Tumor differentiation was assigned by Edmondson-Steiner classification.

Follow-up procedures were described in our previous study. ${ }^{17}$ Overall survival (OS) was defined as the interval between surgery and time of either death or last follow-up. Disease free survival (DFS) was defined as the interval between surgery and time of recurrence, or the date of last follow-up without recurrence. Tumor recurrence was categorized into early and late recurrence using a cutoff value of 2 years. The overall median follow-up time was 70 months (7-79 months).

\section{Statistical Analysis}

SPSS version 20 (IBM Corporation, Armonk, NY, USA) was used to analyze the data. Continuous variables were compared using the Mann-Whitney $U$-test or One-way Anova test. Categorical variables were compared using the $\chi 2$ test or Fisher's exact test. The Spearman rank correlation test was performed to determine any associations between variables. The overall survival and diseasefree survival were computed using the Kaplan-Meier method and were calculated using the Log rank test. A Cox regression hazard model was utilized for the multivariate analysis. To avoid colinearity bias, the lipids and apolipoproteins were preliminarily tested on a first multivariate model that included the individual variables composing the TNM stage. All statistical tests were two-sided and p-values $<0.05$ were interpreted as significant.

\section{Results}

\section{Measurement of Serum Lipids and Apolipoproteins Pattern in Liver Disease}

We first compared the serum lipid and apolipoprotein levels in four different groups. In patients with HCC, the serum level of ApoA1 was statistically lower than that in patients with benign liver disease, but higher than that in liver cirrhosis group, and no significant difference was seen between $\mathrm{HCC}$ and hepatitis groups. In addition, the expression pattern of HDL was similar to ApoA1. Serum ApoE level was significantly higher in HCC group than in chronic liver hepatitis group, whereas it did not differ significantly between the HCC and other two groups. Compared with liver cirrhosis group, the TG and LDL levels were significantly elevated in $\mathrm{HCC}$, liver hepatitis and benign liver disease groups, whereas no significant expression levels were shown among these three groups. Moreover, we did not find any significant differences in serum ApoB or TC levels among all four of these groups. (Figure 1 and Figure S1)

\section{Correlations Between the Lipids and Apolipoproteins Level and Clinical Characteristics}

As shown in Table 1, there were no significant differences in relationships between lipids and apolipoproteins level and clinical background factors such as age, liver cirrhosis, alanine aminotransferase (ALT), albumin (ALB), alphafetoprotein (AFP), or serum total bilirubin (TB). However, ApoA1, ApoE and HDL had close associations with gender. With regard to metabolism profiles, TG and HDL had positive relations with BMI; LDL and ApoB were associated with the glycometabolism indexes as blood glucose (GLU) and hemoglobin A1C (HbA1C), and TG had close relations with $\mathrm{HbA} 1 \mathrm{C}$ and insulin resistance index HOMA2-IR. However, most of lipids and apolipoproteins have no close relations to metabolism parameters in HCC patients. Finally, with respect to tumor related covariates, we revealed that ApoA1 was significantly related to vascular invasion, and LDL was associated with tumor size. However, no significant relations were observed between most lipids and tumor related factors.

\section{Prediction of Tumor Recurrence and Patient Survival in Training Cohort}

We next explored the prognostic significance of lipids and apolipoproteins in patients with HCC. The baseline characteristics of the patients had no significant differences between the training and validation cohorts (Table S1). With the X-tile 3.6.1 software, the optimal cutoff values of each level of lipids and apolipoproteins were set as to separate patients into low and high groups in the training cohort. ${ }^{18}$ The divisions of the validation cohort were also based on these numerical standards. Univariate analysis indicated that lower ApoA1 was significantly correlated with poor prognosis of HCC patients. The cumulative OS and DFS rate of patients in the low ApoA1 group was significantly lower than that of patients in the high ApoA1 group (5-year OS rate: 65 vs 78.8\%, $\mathrm{P}=0.02$; 5-year DFS rate: 45.5 vs $59.6 \%, \mathrm{P}=0.01$ ) (Figure 2 ). In addition, elevated 


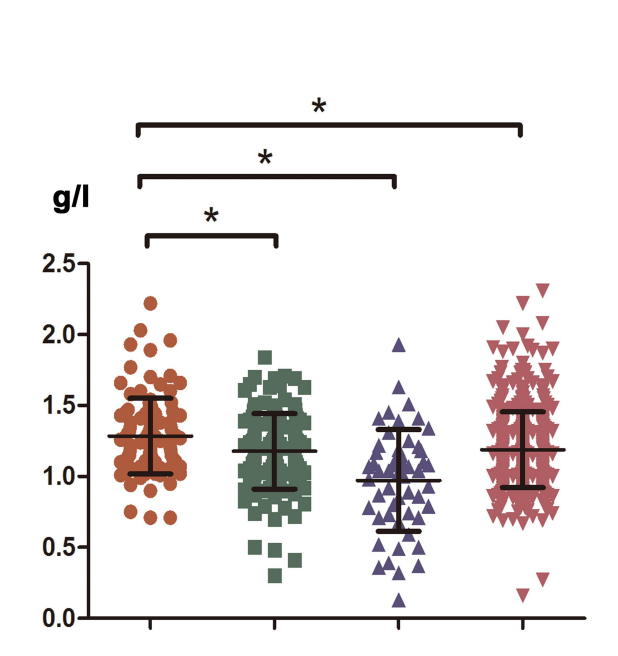

BL CH LC HCC

A

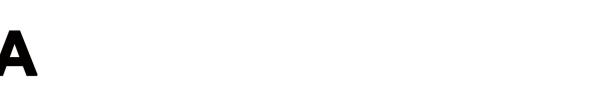

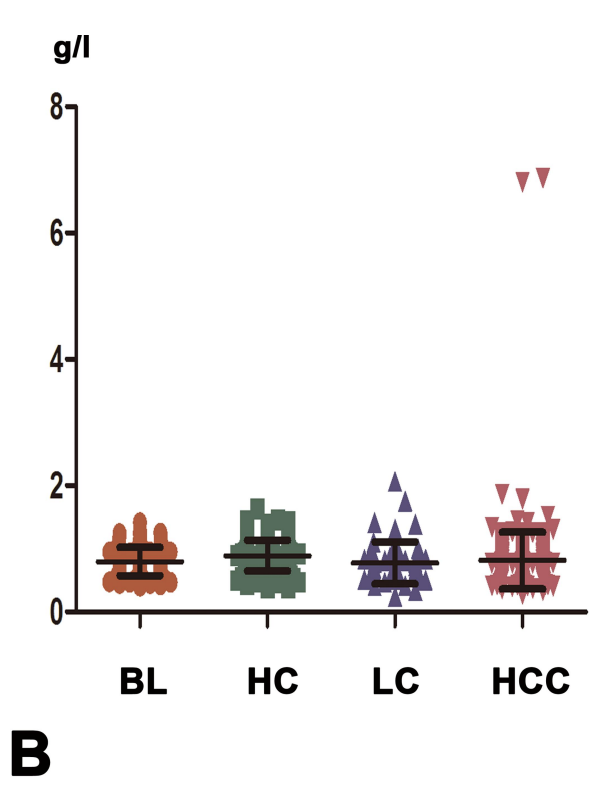

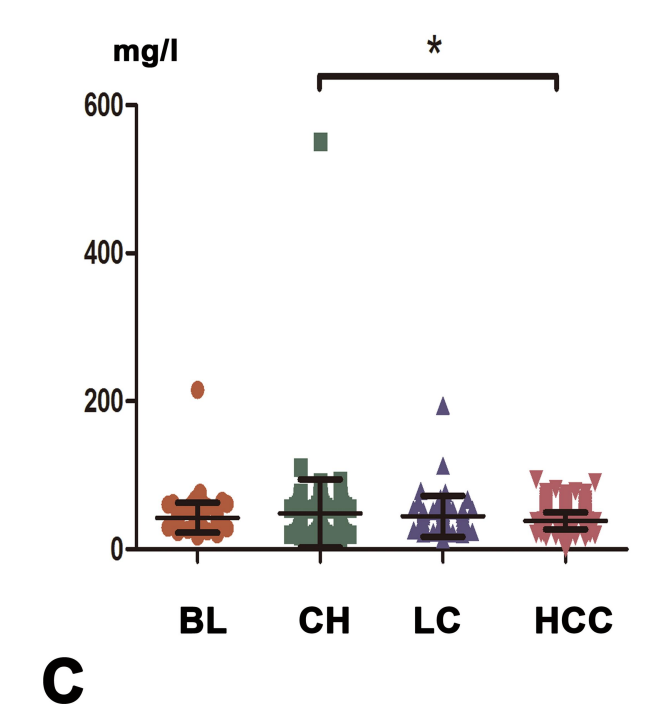

Figure I The blood expression levels of $A p o A I, A p o B$, and $A p o E$ in benign liver disease (BL), chronic hepatitis (CH), liver cirrhosis (LC), and hepatocellular carcinoma (HCC) groups. (A) ApoAI. (B) ApoB. (C) ApoE. * indicates $\mathrm{P}<0.05$.

baseline level of LDL was significantly associated with reduced OS and DFS, while other lipids were not significant predictive factors after univariate analysis (Figure S2 and S3). Multivariate survival analysis conducted by the Cox regression model showed that ApoA1 was confirmed as an independent predictor of both OS and DFS (OS: Hazard ratio (HR): $0.587 ; 95 \%$ confidence interval (CI): 0.352-0.979; $\mathrm{P}=0.04$; DFS: Hazard ratio (HR): $0.607 ; 95 \%$ confidence interval (CI): 0.413-0.893; $\mathrm{P}=0.01$ ). Then a multivariate Cox regression model was used to further assess the independent prognostic ability of the TNM stage. The analysis showed that low ApoA1 remained as an independent unfavorable factor influencing the OS and DFS (OS: HR: 0.517; 95\% CI: 0.309-0.866; $\mathrm{P}=0.01$; DFS: HR: $0.537 ; 95 \% \mathrm{CI}$ : $0.363-0.793 ; \mathrm{P}=0.002$ ) (Table 2 and Table $\mathrm{S} 2$ ).

\section{Prognostic Value of Lipids and Apolipoproteins Level in the Validation Cohort}

Lipids and apolipoproteins were further assessed for their prognostic power in an independent cohort of 229 patients. We also found that HCC patients with lower serum ApoA1 
levels had significantly worse prognosis than those with higher serum ApoA1 levels. In univariate analysis of OS, ApoA1 and ApoE were univariate predictors of OS $(\mathrm{P}=0.01 ; \mathrm{P}=0.02$, respectively) (Figure 2 and Figure $\mathrm{S} 4$ ). Cox regression analysis demonstrated that lower serum ApoA1 level was an independent indicator of OS (HR: 0.418; 95\% CI: 0.234-0.747; $\mathrm{P}=0.003$ ). In addition, a higher serum ApoE level was also an independent risk factor for OS (HR:1.897; 95\% CI: 1.123-3.204; $\mathrm{P}=0.02$ ). In univariate analysis of DFS, lower ApoA1 level was a significant factor of poor prognosis $(\mathrm{P}=0.007)$, and $\mathrm{Cox}$ regression analysis showed that ApoA1 remained as an independent prognostic factor for DFS (HR:0.609; 95\% CI: 0.409-0.908; $\mathrm{P}=0.02$ ) (Table S3 and Table S4). However, other lipids and apolipoproteins including CHOL, TG, HDL, LDL and ApoB did not show any statistically significant associations with OS and DFS (Figure S4 and S5).

\section{Serum ApoA I Levels Correlate with Early Recurrence in the Training and Validation Cohort}

Furthermore, the prognostic significance of serum ApoA1 levels within $\alpha$-fetoprotein (AFP) $\leq 400 \mathrm{ng} / \mathrm{mL}$ or/and early recurrence subgroups was investigated. Among patients with an AFP concentration of $\leq 400 \mathrm{ng} / \mathrm{mL}$, the OS and DFS rate in low ApoA1 patients were significantly lower than that in high ApoA1 patients in the training cohort (OS: $68.3 \%$ vs $83.1 \%, \mathrm{P}=0.006$; DFS: $49 \%$ vs $66.7 \%$, $\mathrm{P}=0.01$, respectively). In accordance with prognosis value of ApoA1 in the training cohort, ApoA1 remained associated with the OS and DFS rate in the lower AFP subgroup in the validation cohort (OS: $72.2 \%$ vs $86.4 \%$, $\mathrm{P}=0.025$; DFS: $47.4 \%$ vs $62.1 \%, \mathrm{P}=0.04$, respectively).

In addition, it is worth noting that as for early recurrence in the training cohort, serum ApoA1 level was significantly associated with it in the univariate analysis. In further multivariate analysis, the serum ApoA1 level was an independent indicator for early recurrence (HR: 0.51; 95\% CI: 0.297-0.875; $\mathrm{P}=0.02$ ). Similar results were confirmed in the validation cohorts, lower ApoA-1 was an independent predictor of early recurrence (HR, 0.478; 95\% CI: $0.278-0.881 ; \mathrm{P}=0.008$ ) (Figure 3, Table 3 and Table

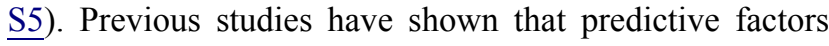
for early recurrence are mainly tumor-related clinicopathologic factors (ie, tumor size, tumor number, tumor marker, microscopic vascular invasion). ${ }^{3,19}$ The results of the present study also show that early recurrence was associated at multivariate analysis with larger tumor size, vascular invasion, and advanced TNM stage. Further stratification analyses were conducted in subgroups based on AFP, tumor size, number, vascular invasion, and TNM stage. We found that low ApoA1 was remarkably related to the early recurrence of patients with an AFP concentration of $\leq 400 \mu \mathrm{g} / \mathrm{L}$ and tumor size ranging from $2 \mathrm{~cm}$ to $5 \mathrm{~cm}$ and TNM stage I and solitary tumor in both the training and validation cohort. By contrast, no significant associations were observed between ApoA1 level and early recurrence rate in vascular invasion negative subgroups (Figure S7 and $\underline{\mathrm{S} 8}$ ).

\section{Discussion}

To date the association between lipids and apolipoproteins and HCC has not been thoroughly investigated and there is scanty data comprehensively reported concerning their clinical significance in relation to HCC. In this study, we analyzed the serum lipid and apolipoprotein levels in patients with benign lesions, chronic hepatitis, liver cirrhosis and $\mathrm{HCC}$, and found that the majority of circulating lipid and apolipoprotein levels in chronic liver disease and HCC were slightly to significantly decreased as compared with those in benign lesion. This result was in accordance with some previous studies. , $^{9,10,13,20}$

As we know, the currently available serological biomarkers such as AFP are insufficiently sensitive or specific for use in diagnostic assays, ${ }^{21,22}$ thus novel serum markers capable of precise detection of $\mathrm{HCC}$ at an early stage and accurate monitoring over a long period for $\mathrm{HCC}$ are needed. Proteomic technology has revealed certain apolipoproteins can distinguish between serum from patients with cancer and healthy control subjects with high sensitivity and specificity, for instance, ApoA1. Mustafa et al have discovered proteomic alterations of ApoA1 in HCC serum samples, a decreased level of ApoA1 relative to that in HCV. Similar to the above result, Liu et al found a great discriminatory value of ApoA1 between the two patient populations with $\mathrm{HCC}$ and liver cirrhosis, and suggested ApoA1 had a diagnostic ability in detecting $\mathrm{HCC}^{23}$ According to above-mentioned results, it seems that the conclusion on ApoA1 as an effective diagnosis biomarker for HCC could be drawn. Although our results showed that the majority lipids and apolipoproteins levels have apparently changed between HCC and normal benign groups, no significant differential expression patterns of lipids and apolipoproteins were observed between chronic liver 
Table I Relationship Between Lipids and Apolipoproteins Level and Clinical Variables of HCC Patients

\begin{tabular}{|c|c|c|c|c|c|c|c|c|c|}
\hline \multicolumn{2}{|l|}{ Variables } & \multicolumn{2}{|l|}{ ApoAl } & \multirow[t]{3}{*}{ p value } & \multicolumn{2}{|l|}{ ApoB } & \multirow[t]{3}{*}{$p$ value } & \multicolumn{2}{|l|}{ ApoE } \\
\hline & & \multirow{2}{*}{$\begin{array}{l}\text { low } \\
\mathrm{n}=\mathbf{2 8 2}\end{array}$} & \multirow{2}{*}{$\begin{array}{l}\text { high } \\
n=189\end{array}$} & & \multirow{2}{*}{$\begin{array}{l}\text { low } \\
n=345\end{array}$} & \multirow{2}{*}{$\begin{array}{l}\text { high } \\
n=126\end{array}$} & & low & high \\
\hline & & & & & & & & $\mathrm{n}=253$ & $n=218$ \\
\hline Gender & $\begin{array}{l}\text { Male } \\
\text { Female }\end{array}$ & $\begin{array}{l}258 \\
24\end{array}$ & $\begin{array}{l}140 \\
49\end{array}$ & 0 & $\begin{array}{l}290 \\
55\end{array}$ & $\begin{array}{l}108 \\
18\end{array}$ & 0.66 & $\begin{array}{l}222 \\
31\end{array}$ & $\begin{array}{l}176 \\
42\end{array}$ \\
\hline Age(years) & $\begin{array}{l}<55 \\
\geq 55\end{array}$ & $\begin{array}{l}152 \\
130\end{array}$ & $\begin{array}{l}87 \\
102\end{array}$ & 0.09 & $\begin{array}{l}182 \\
163\end{array}$ & $\begin{array}{l}57 \\
69\end{array}$ & 0.15 & $\begin{array}{l}130 \\
123\end{array}$ & $\begin{array}{l}109 \\
109\end{array}$ \\
\hline liver cirrhosis & $\begin{array}{l}\text { absence } \\
\text { present }\end{array}$ & $\begin{array}{l}76 \\
206\end{array}$ & $\begin{array}{l}51 \\
138\end{array}$ & 1 & $\begin{array}{l}86 \\
259\end{array}$ & $\begin{array}{l}41 \\
85\end{array}$ & 0.1 & $\begin{array}{l}62 \\
191\end{array}$ & $\begin{array}{l}65 \\
153\end{array}$ \\
\hline $\begin{array}{l}\text { Hepatitis } \\
\text { history }\end{array}$ & $\begin{array}{l}\text { absence } \\
\text { present }\end{array}$ & $\begin{array}{l}92 \\
190\end{array}$ & $\begin{array}{l}47 \\
142\end{array}$ & 0.08 & $\begin{array}{l}98 \\
247\end{array}$ & $\begin{array}{l}41 \\
85\end{array}$ & 0.43 & $\begin{array}{l}74 \\
179\end{array}$ & $\begin{array}{l}65 \\
153\end{array}$ \\
\hline BMI & $\begin{array}{l}<23.5 \\
\geq 23.5\end{array}$ & $\begin{array}{l}14 \mid \\
141\end{array}$ & $\begin{array}{l}90 \\
99\end{array}$ & 0.64 & $\begin{array}{l}175 \\
170\end{array}$ & $\begin{array}{l}56 \\
70\end{array}$ & 0.25 & $\begin{array}{l}132 \\
121\end{array}$ & $\begin{array}{l}99 \\
119\end{array}$ \\
\hline GLU & $\begin{array}{l}<5.6 \\
\geq 5.6\end{array}$ & $\begin{array}{l}228 \\
54\end{array}$ & $\begin{array}{l}146 \\
43\end{array}$ & 0.35 & $\begin{array}{l}284 \\
61\end{array}$ & $\begin{array}{l}90 \\
36\end{array}$ & 0.01 & $\begin{array}{l}209 \\
44\end{array}$ & $\begin{array}{l}165 \\
53\end{array}$ \\
\hline APLB & $\begin{array}{l}<0.25 \\
\geq 0.25\end{array}$ & $\begin{array}{l}212 \\
70\end{array}$ & $\begin{array}{l}130 \\
59\end{array}$ & 0.13 & $\begin{array}{l}265 \\
80\end{array}$ & $\begin{array}{l}77 \\
49\end{array}$ & 0 & $\begin{array}{l}200 \\
53\end{array}$ & $\begin{array}{l}142 \\
76\end{array}$ \\
\hline ALT & $\begin{array}{l}\leq 50 \\
>50\end{array}$ & $\begin{array}{l}224 \\
58\end{array}$ & $\begin{array}{l}146 \\
43\end{array}$ & 0.57 & $\begin{array}{l}270 \\
75\end{array}$ & $\begin{array}{l}100 \\
26\end{array}$ & 0.8 & $\begin{array}{l}210 \\
43\end{array}$ & $\begin{array}{l}160 \\
58\end{array}$ \\
\hline TB & $\begin{array}{l}\leq 20 \\
>20\end{array}$ & $\begin{array}{l}258 \\
24\end{array}$ & $\begin{array}{l}171 \\
18\end{array}$ & 0.71 & $\begin{array}{l}314 \\
31\end{array}$ & $\begin{array}{l}115 \\
11\end{array}$ & 0.93 & $\begin{array}{l}233 \\
20\end{array}$ & $\begin{array}{l}196 \\
22\end{array}$ \\
\hline AFP & $\begin{array}{l}\leq 400 \\
>400\end{array}$ & $\begin{array}{l}201 \\
81\end{array}$ & $\begin{array}{l}138 \\
51\end{array}$ & 0.68 & $\begin{array}{l}244 \\
101\end{array}$ & $\begin{array}{l}95 \\
31\end{array}$ & 0.32 & $\begin{array}{l}181 \\
72\end{array}$ & $\begin{array}{l}158 \\
60\end{array}$ \\
\hline ALB & $\begin{array}{l}\leq 40 \\
>40\end{array}$ & $\begin{array}{l}90 \\
192\end{array}$ & $\begin{array}{l}61 \\
128\end{array}$ & 0.94 & $\begin{array}{l}119 \\
226\end{array}$ & $\begin{array}{l}32 \\
94\end{array}$ & 0.06 & $\begin{array}{l}77 \\
176\end{array}$ & $\begin{array}{l}74 \\
144\end{array}$ \\
\hline GGT & $\begin{array}{l}\leq 60 \\
>60\end{array}$ & $\begin{array}{l}175 \\
107\end{array}$ & $\begin{array}{l}108 \\
81\end{array}$ & 0.29 & $\begin{array}{l}219 \\
126\end{array}$ & $\begin{array}{l}64 \\
62\end{array}$ & 0.01 & $\begin{array}{l}165 \\
88\end{array}$ & $\begin{array}{l}118 \\
100\end{array}$ \\
\hline $\mathrm{HbAIC}$ & $\begin{array}{l}<6 \\
\geq 6\end{array}$ & $\begin{array}{l}232 \\
50\end{array}$ & $\begin{array}{l}154 \\
35\end{array}$ & 0.83 & $\begin{array}{l}298 \\
47\end{array}$ & $\begin{array}{l}88 \\
38\end{array}$ & $<0.001$ & $\begin{array}{l}212 \\
41\end{array}$ & $\begin{array}{l}174 \\
44\end{array}$ \\
\hline HOMA2-IR & $\begin{array}{l}<26.9 \\
\geq 26.9\end{array}$ & $\begin{array}{l}262 \\
20\end{array}$ & $\begin{array}{l}175 \\
14\end{array}$ & 0.9 & $\begin{array}{l}318 \\
27\end{array}$ & $\begin{array}{l}119 \\
7\end{array}$ & 0.4 & $\begin{array}{l}237 \\
16\end{array}$ & $\begin{array}{l}200 \\
18\end{array}$ \\
\hline CRP & $\begin{array}{l}<3 \\
\geq 3\end{array}$ & $\begin{array}{l}198 \\
84\end{array}$ & $\begin{array}{l}144 \\
45\end{array}$ & 0.15 & $\begin{array}{l}267 \\
78\end{array}$ & $\begin{array}{l}75 \\
51\end{array}$ & $<0.001$ & $\begin{array}{l}188 \\
65\end{array}$ & $\begin{array}{l}154 \\
64\end{array}$ \\
\hline Tumor size & $\begin{array}{l}\leq 5 \mathrm{~cm} \\
>5 \mathrm{~cm}\end{array}$ & $\begin{array}{l}184 \\
98\end{array}$ & $\begin{array}{l}133 \\
56\end{array}$ & 0.27 & $\begin{array}{l}237 \\
108\end{array}$ & $\begin{array}{l}80 \\
46\end{array}$ & 0.32 & $\begin{array}{l}180 \\
73\end{array}$ & $\begin{array}{l}137 \\
81\end{array}$ \\
\hline Tumor number & $\begin{array}{l}\text { Single } \\
\text { Multiple }\end{array}$ & $\begin{array}{l}245 \\
37\end{array}$ & $\begin{array}{l}162 \\
27\end{array}$ & 0.72 & $\begin{array}{l}303 \\
42\end{array}$ & $\begin{array}{l}104 \\
22\end{array}$ & 0.14 & $\begin{array}{l}222 \\
31\end{array}$ & $\begin{array}{l}185 \\
33\end{array}$ \\
\hline Tumor capsule & $\begin{array}{l}\text { complete } \\
\text { None }\end{array}$ & $\begin{array}{l}180 \\
102\end{array}$ & $\begin{array}{l}123 \\
66\end{array}$ & 0.78 & $\begin{array}{l}219 \\
126\end{array}$ & $\begin{array}{l}84 \\
42\end{array}$ & 0.52 & $\begin{array}{l}160 \\
93\end{array}$ & $\begin{array}{l}143 \\
75\end{array}$ \\
\hline Vascular invasion & $\begin{array}{l}\text { absence } \\
\text { present }\end{array}$ & $\begin{array}{l}198 \\
84\end{array}$ & $\begin{array}{l}152 \\
37\end{array}$ & 0.01 & $\begin{array}{l}260 \\
85\end{array}$ & $\begin{array}{l}90 \\
36\end{array}$ & 0.39 & $\begin{array}{l}190 \\
63\end{array}$ & $\begin{array}{l}160 \\
58\end{array}$ \\
\hline $\begin{array}{l}\text { Edmondson } \\
\text { grade }\end{array}$ & $\begin{array}{l}\text { I-II } \\
\text { III-IV }\end{array}$ & $\begin{array}{l}202 \\
80\end{array}$ & $\begin{array}{l}133 \\
56\end{array}$ & 0.77 & $\begin{array}{l}239 \\
106\end{array}$ & $\begin{array}{l}96 \\
30\end{array}$ & 0.14 & $\begin{array}{l}178 \\
75\end{array}$ & $\begin{array}{l}157 \\
61\end{array}$ \\
\hline
\end{tabular}




\begin{tabular}{|c|c|c|c|c|c|c|c|c|c|c|c|c|}
\hline \multirow[t]{3}{*}{$p$ value } & \multicolumn{2}{|l|}{ TC } & \multirow[t]{3}{*}{ p value } & \multicolumn{2}{|l|}{ TG } & \multirow[t]{3}{*}{$p$ value } & \multicolumn{2}{|l|}{ HDL } & \multirow[t]{3}{*}{$p$ value } & \multicolumn{2}{|l|}{ LDL } & \multirow[t]{3}{*}{ P value } \\
\hline & low & high & & low & high & & low & high & & low & high & \\
\hline & $n=420$ & $n=51$ & & $n=377$ & $n=94$ & & $n=279$ & $n=192$ & & $n=166$ & $n=305$ & \\
\hline 0.04 & $\begin{array}{l}355 \\
65\end{array}$ & $\begin{array}{l}43 \\
8\end{array}$ & 0.97 & $\begin{array}{l}316 \\
61\end{array}$ & $\begin{array}{l}82 \\
12\end{array}$ & 0.41 & $\begin{array}{l}222 \\
57\end{array}$ & $\begin{array}{l}176 \\
16\end{array}$ & $<0.001$ & $\begin{array}{l}135 \\
31\end{array}$ & $\begin{array}{l}263 \\
42\end{array}$ & 0.16 \\
\hline 0.77 & $\begin{array}{l}214 \\
206\end{array}$ & $\begin{array}{l}25 \\
26\end{array}$ & 0.79 & $\begin{array}{l}192 \\
185\end{array}$ & $\begin{array}{l}47 \\
47\end{array}$ & 0.87 & $\begin{array}{l}132 \\
147\end{array}$ & $\begin{array}{l}107 \\
85\end{array}$ & 0.07 & $\begin{array}{l}86 \\
80\end{array}$ & $\begin{array}{l}153 \\
152\end{array}$ & 0.73 \\
\hline 0.21 & $\begin{array}{l}109 \\
311\end{array}$ & $\begin{array}{l}18 \\
33\end{array}$ & 0.18 & $\begin{array}{l}93 \\
284\end{array}$ & $\begin{array}{l}34 \\
60\end{array}$ & 0.03 & $\begin{array}{l}68 \\
211\end{array}$ & $\begin{array}{l}59 \\
133\end{array}$ & 0.14 & $\begin{array}{l}39 \\
127\end{array}$ & $\begin{array}{l}88 \\
217\end{array}$ & 0.23 \\
\hline 0.92 & $\begin{array}{l}125 \\
295\end{array}$ & $\begin{array}{l}14 \\
37\end{array}$ & 0.75 & $\begin{array}{l}103 \\
274\end{array}$ & $\begin{array}{l}36 \\
58\end{array}$ & 0.04 & $\begin{array}{l}80 \\
199\end{array}$ & $\begin{array}{l}59 \\
133\end{array}$ & 0.68 & $\begin{array}{l}51 \\
115\end{array}$ & $\begin{array}{l}88 \\
217\end{array}$ & 0.67 \\
\hline 0.17 & $\begin{array}{l}207 \\
213\end{array}$ & $\begin{array}{l}24 \\
27\end{array}$ & 0.77 & $\begin{array}{l}202 \\
175\end{array}$ & $\begin{array}{l}29 \\
65\end{array}$ & $<0.001$ & $\begin{array}{l}149 \\
130\end{array}$ & $\begin{array}{l}82 \\
110\end{array}$ & 0.03 & $\begin{array}{l}87 \\
79\end{array}$ & $\begin{array}{l}144 \\
161\end{array}$ & 0.29 \\
\hline 0.07 & $\begin{array}{l}338 \\
82\end{array}$ & $\begin{array}{l}36 \\
15\end{array}$ & 0.14 & $\begin{array}{l}302 \\
75\end{array}$ & $\begin{array}{l}72 \\
22\end{array}$ & 0.48 & $\begin{array}{l}224 \\
55\end{array}$ & $\begin{array}{l}150 \\
42\end{array}$ & 0.64 & $\begin{array}{l}141 \\
25\end{array}$ & $\begin{array}{l}233 \\
72\end{array}$ & 0.03 \\
\hline 0 & $\begin{array}{l}313 \\
107\end{array}$ & $\begin{array}{l}29 \\
22\end{array}$ & 0.01 & $\begin{array}{l}296 \\
81\end{array}$ & $\begin{array}{l}46 \\
48\end{array}$ & $<0.001$ & $\begin{array}{l}208 \\
71\end{array}$ & $\begin{array}{l}134 \\
58\end{array}$ & 0.26 & $\begin{array}{l}136 \\
30\end{array}$ & $\begin{array}{l}206 \\
99\end{array}$ & 0.001 \\
\hline 0.01 & $\begin{array}{l}333 \\
87\end{array}$ & $\begin{array}{l}37 \\
14\end{array}$ & 0.27 & $\begin{array}{l}301 \\
76\end{array}$ & $\begin{array}{l}69 \\
25\end{array}$ & 0.17 & $\begin{array}{l}212 \\
67\end{array}$ & $\begin{array}{l}158 \\
34\end{array}$ & 0.1 & $\begin{array}{l}132 \\
34\end{array}$ & $\begin{array}{l}238 \\
67\end{array}$ & 0.71 \\
\hline 0.41 & $\begin{array}{l}380 \\
40\end{array}$ & $\begin{array}{l}49 \\
2\end{array}$ & 0.19 & $\begin{array}{l}344 \\
33\end{array}$ & $\begin{array}{l}85 \\
9\end{array}$ & 0.8 & $\begin{array}{l}255 \\
24\end{array}$ & $\begin{array}{l}174 \\
18\end{array}$ & 0.77 & $\begin{array}{l}149 \\
17\end{array}$ & $\begin{array}{l}280 \\
25\end{array}$ & 0.46 \\
\hline 0.82 & $\begin{array}{l}304 \\
116\end{array}$ & $\begin{array}{l}35 \\
16\end{array}$ & 0.57 & $\begin{array}{l}262 \\
115\end{array}$ & $\begin{array}{l}77 \\
17\end{array}$ & 0.02 & $\begin{array}{l}193 \\
86\end{array}$ & $\begin{array}{l}146 \\
46\end{array}$ & 0.1 & $\begin{array}{l}117 \\
49\end{array}$ & $\begin{array}{l}222 \\
83\end{array}$ & 0.6 \\
\hline 0.42 & $\begin{array}{l}136 \\
284\end{array}$ & $\begin{array}{l}15 \\
36\end{array}$ & 0.67 & $\begin{array}{l}123 \\
254\end{array}$ & $\begin{array}{l}28 \\
66\end{array}$ & 0.6 & $\begin{array}{l}98 \\
181\end{array}$ & $\begin{array}{l}53 \\
139\end{array}$ & 0.09 & $\begin{array}{l}59 \\
107\end{array}$ & $\begin{array}{l}92 \\
213\end{array}$ & 0.23 \\
\hline 0.01 & $\begin{array}{l}255 \\
165\end{array}$ & $\begin{array}{l}28 \\
23\end{array}$ & 0.42 & $\begin{array}{l}239 \\
138\end{array}$ & $\begin{array}{l}44 \\
50\end{array}$ & 0 & $\begin{array}{l}157 \\
122\end{array}$ & $\begin{array}{l}126 \\
66\end{array}$ & 0.04 & $\begin{array}{l}114 \\
52\end{array}$ & $\begin{array}{l}169 \\
136\end{array}$ & 0.01 \\
\hline 0.26 & $\begin{array}{l}348 \\
72\end{array}$ & $\begin{array}{l}38 \\
13\end{array}$ & 0.14 & $\begin{array}{l}317 \\
60\end{array}$ & $\begin{array}{l}69 \\
25\end{array}$ & 0.02 & $\begin{array}{l}231 \\
48\end{array}$ & $\begin{array}{l}155 \\
37\end{array}$ & 0.57 & $\begin{array}{l}146 \\
20\end{array}$ & $\begin{array}{l}240 \\
65\end{array}$ & 0.01 \\
\hline 0.42 & $\begin{array}{l}390 \\
47\end{array}$ & $\begin{array}{l}30 \\
4\end{array}$ & 0.86 & $\begin{array}{l}355 \\
22\end{array}$ & $\begin{array}{l}82 \\
12\end{array}$ & 0.02 & $\begin{array}{l}261 \\
18\end{array}$ & $\begin{array}{l}176 \\
16\end{array}$ & 0.44 & $\begin{array}{l}155 \\
11\end{array}$ & $\begin{array}{l}282 \\
23\end{array}$ & 0.71 \\
\hline 0.37 & $\begin{array}{l}306 \\
114\end{array}$ & $\begin{array}{l}36 \\
15\end{array}$ & 0.73 & $\begin{array}{l}274 \\
103\end{array}$ & $\begin{array}{l}68 \\
26\end{array}$ & 0.95 & $\begin{array}{l}206 \\
73\end{array}$ & $\begin{array}{l}136 \\
56\end{array}$ & 0.47 & $\begin{array}{l}134 \\
32\end{array}$ & $\begin{array}{l}208 \\
97\end{array}$ & 0.004 \\
\hline 0.06 & $\begin{array}{l}284 \\
136\end{array}$ & $\begin{array}{l}33 \\
18\end{array}$ & 0.86 & $\begin{array}{l}250 \\
127\end{array}$ & $\begin{array}{l}67 \\
27\end{array}$ & 0.39 & $\begin{array}{l}182 \\
97\end{array}$ & $\begin{array}{l}135 \\
57\end{array}$ & 0.27 & $\begin{array}{l}124 \\
42\end{array}$ & $\begin{array}{l}193 \\
112\end{array}$ & 0.01 \\
\hline 0.36 & $\begin{array}{l}367 \\
53\end{array}$ & $\begin{array}{l}40 \\
11\end{array}$ & 0.08 & $\begin{array}{l}328 \\
49\end{array}$ & $\begin{array}{l}79 \\
15\end{array}$ & 0.45 & $\begin{array}{l}237 \\
42\end{array}$ & $\begin{array}{l}170 \\
22\end{array}$ & 0.26 & $\begin{array}{l}138 \\
28\end{array}$ & $\begin{array}{l}269 \\
36\end{array}$ & 0.16 \\
\hline 0.6 & $\begin{array}{l}269 \\
151\end{array}$ & $\begin{array}{l}34 \\
17\end{array}$ & 0.71 & $\begin{array}{l}240 \\
137\end{array}$ & $\begin{array}{l}63 \\
31\end{array}$ & 0.54 & $\begin{array}{l}176 \\
103\end{array}$ & $\begin{array}{l}127 \\
65\end{array}$ & 0.5 & $\begin{array}{l}98 \\
68\end{array}$ & $\begin{array}{l}205 \\
100\end{array}$ & 0.08 \\
\hline 0.67 & $\begin{array}{l}314 \\
106\end{array}$ & $\begin{array}{l}36 \\
15\end{array}$ & 0.52 & $\begin{array}{l}274 \\
103\end{array}$ & $\begin{array}{l}76 \\
18\end{array}$ & 0.11 & $\begin{array}{l}204 \\
75\end{array}$ & $\begin{array}{l}146 \\
46\end{array}$ & 0.48 & $\begin{array}{l}130 \\
36\end{array}$ & $\begin{array}{l}220 \\
85\end{array}$ & 0.14 \\
\hline 0.69 & $\begin{array}{l}295 \\
125\end{array}$ & $\begin{array}{l}40 \\
11\end{array}$ & 0.22 & $\begin{array}{l}263 \\
114\end{array}$ & $\begin{array}{l}72 \\
22\end{array}$ & 0.19 & $\begin{array}{l}188 \\
91\end{array}$ & $\begin{array}{l}147 \\
45\end{array}$ & 0.03 & $\begin{array}{l}112 \\
54\end{array}$ & $\begin{array}{l}223 \\
82\end{array}$ & 0.2 \\
\hline
\end{tabular}


Table I (Continued).

\begin{tabular}{|c|c|c|c|c|c|c|c|c|c|}
\hline \multicolumn{2}{|l|}{ Variables } & \multicolumn{2}{|l|}{ ApoAl } & \multirow[t]{3}{*}{$\mathrm{p}$ value } & \multicolumn{2}{|l|}{ ApoB } & \multirow[t]{3}{*}{$\mathrm{p}$ value } & \multicolumn{2}{|l|}{ ApoE } \\
\hline & & low & high & & low & high & & low & high \\
\hline & & $n=282$ & $n=189$ & & $n=345$ & $n=126$ & & $n=253$ & $n=218$ \\
\hline TNM stage & $\begin{array}{l}\text { I } \\
\text { II } \\
\text { III }\end{array}$ & $\begin{array}{l}230 \\
28 \\
24\end{array}$ & $\begin{array}{l}154 \\
18 \\
17\end{array}$ & 0.98 & $\begin{array}{l}286 \\
31 \\
28\end{array}$ & $\begin{array}{l}98 \\
15 \\
13\end{array}$ & 0.45 & $\begin{array}{l}211 \\
25 \\
17\end{array}$ & $\begin{array}{l}173 \\
21 \\
24\end{array}$ \\
\hline
\end{tabular}

Abbreviations: BMI, body mass index; GLU, glucose; APLB, prealbumin; ALT, alanine aminotransferase; TB, total bilirubin; AFP, alpha-fetoprotein; ALB, prealbumin; GGT, glutamyl transpeptidase; HbAIC, hemoglobin AIC; CRP, C reactive protein; TC, total cholesterol; TG, triglycerides; HDL, high-density lipoprotein; LDL, low-density lipoprotein.

hepatitis and HCC in our study. Furthermore, the circulating ApoA1, HDL and LDL levels were strongly lower in liver cirrhosis group as compared to HCC group, and this result was not contradictory with previous studies. ${ }^{10,13,24}$ A possible explanation for this is that the majority of previous studies included patients with liver cancer at
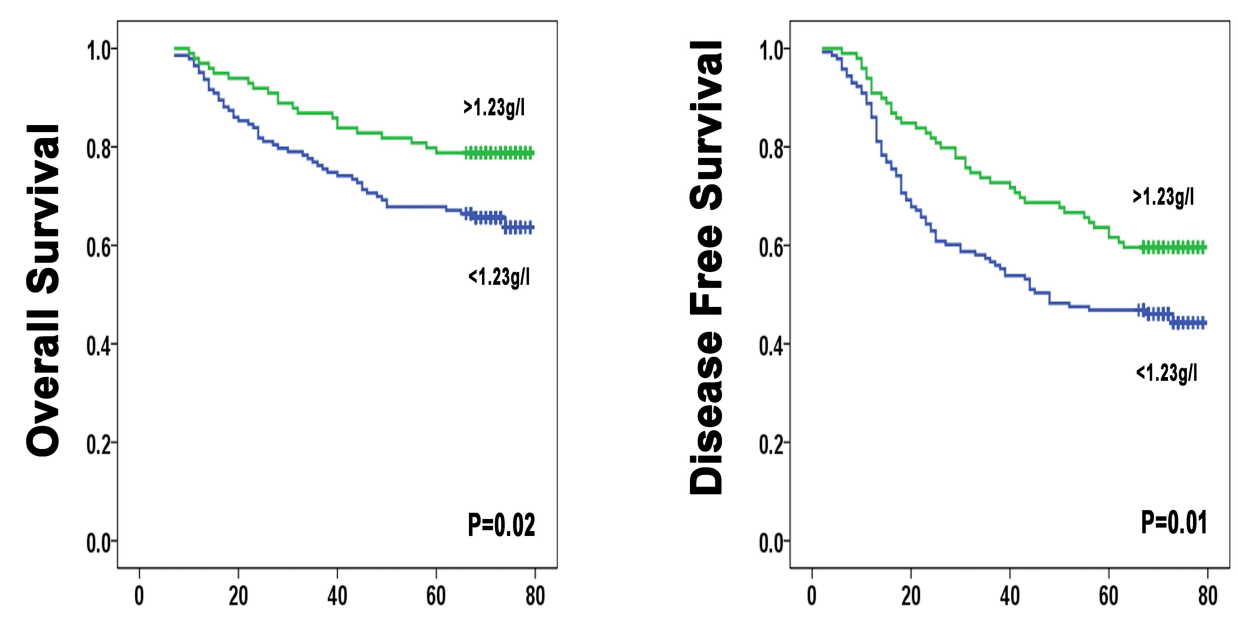

\section{A Time(month)}


D Time(month)

Figure 2 Low ApoAI level is significantly associated with poorer patient survival. Low ApoAI level is significantly associated with poorer overall survival (A) and disease-free survival (B), in a test cohort of $242 \mathrm{HCC}$ patients. Low ApoAI level is significantly associated with poorer overall survival (C) and recurrence-free survival (D), in a validation cohort of $229 \mathrm{HCC}$ patients. 


\begin{tabular}{|c|c|c|c|c|c|c|c|c|c|c|c|c|}
\hline \multirow[t]{3}{*}{$p$ value } & \multicolumn{2}{|l|}{ TC } & \multirow[t]{3}{*}{ P value } & \multicolumn{2}{|l|}{ TG } & \multirow[t]{3}{*}{$p$ value } & \multicolumn{2}{|l|}{ HDL } & \multirow[t]{3}{*}{$p$ value } & \multicolumn{2}{|l|}{ LDL } & \multirow[t]{3}{*}{ p value } \\
\hline & low & high & & low & high & & low & high & & low & high & \\
\hline & $n=420$ & $n=51$ & & $n=377$ & $\mathrm{n}=94$ & & $\mathrm{n}=279$ & $n=192$ & & $n=166$ & $\mathrm{n}=305$ & \\
\hline 0.26 & $\begin{array}{l}346 \\
39 \\
35\end{array}$ & $\begin{array}{l}38 \\
7 \\
6\end{array}$ & 0.39 & $\begin{array}{l}308 \\
34 \\
35\end{array}$ & $\begin{array}{l}76 \\
12 \\
6\end{array}$ & 0.4 & $\begin{array}{l}222 \\
31 \\
26\end{array}$ & $\begin{array}{l}162 \\
15 \\
15\end{array}$ & 0.39 & $\begin{array}{l}133 \\
20 \\
13\end{array}$ & $\begin{array}{l}251 \\
26 \\
28\end{array}$ & 0.44 \\
\hline
\end{tabular}

more advanced stages, accompanied with more severe liver dysfunction, inevitably leading to a lower level of lipids and apolipoproteins compared with our results. Collectively, we considered that ApoA1 was not a sensitive or specific biomarker enough to separate HCC from chronic liver diseases. In addition, some data have suggested ApoA1 could provide diagnostic utility to distinguish patients with bladder cancer from healthy controls, as well as gastric and breast cancer. The more important concern is that these results emphasize a fact that changes of lipid and apolipoprotein profiles may imply the relationship with the presence and/or risk of

Table 2 Univariate and Multivariate Analyses of Prognostic Factors in HCC in Training Cohort

\begin{tabular}{|c|c|c|c|c|c|c|}
\hline \multirow[t]{3}{*}{ Variables } & \multicolumn{3}{|l|}{ OS } & \multicolumn{3}{|l|}{ DFS } \\
\hline & \multirow{2}{*}{$\begin{array}{l}\text { Univariate } \\
\mathbf{P}\end{array}$} & \multicolumn{2}{|l|}{ Multivariate } & \multirow{2}{*}{$\begin{array}{l}\text { Univariate } \\
\mathbf{P}\end{array}$} & \multicolumn{2}{|l|}{ Multivariate } \\
\hline & & HR (95\% Cl) & $\mathbf{P}$ & & HR $(95 \% \mathrm{CI})$ & $\mathbf{P}$ \\
\hline Gender & 0.93 & & & 0.57 & & \\
\hline Age(years) & 0.46 & & & 0.08 & & \\
\hline liver cirrhosis & 0.59 & & & 0.45 & & \\
\hline Hepatitis history & $0.8 \mathrm{I}$ & & & 0.15 & & \\
\hline BMI & 0.18 & & & 0.11 & & \\
\hline GLU & 0.59 & & & 0.7 & & \\
\hline APLB & 0.08 & & & 0.12 & & \\
\hline ALT & 0.89 & & & 0.55 & & \\
\hline TB & 0.25 & & & 0.61 & & \\
\hline AFP & 0.002 & $1.687(I .028-2.77 I)$ & 0.04 & 0.004 & & \\
\hline ALB & 0.56 & & & 0.71 & & \\
\hline GGT & 0.01 & & & 0.004 & & \\
\hline $\mathrm{CHOL}$ & 0.56 & & & 0.51 & & \\
\hline TG & 0.24 & & & 0.32 & & \\
\hline HDL & 0.36 & & & 0.57 & & \\
\hline LDL & 0.01 & $1.868(1.036-3.368)$ & 0.04 & 0.045 & & \\
\hline Tumor size & $<0.001$ & $2.216(I .379-3.56 \mid)$ & 0.001 & $<0.001$ & $1.974(1.363-2.862)$ & $<0.001$ \\
\hline Tumor number & 0.08 & & & 0.09 & & \\
\hline Tumor capsule & 0.04 & & & 0.26 & & \\
\hline Vascular invasion & $<0.001$ & $2.502(I .542-4.06 \mid)$ & $<0.001$ & $<0.001$ & $1.997(1.336-2.984)$ & 0.001 \\
\hline Edmondson grade & 0.054 & & & 0.007 & $1.458(0.98-2.17)$ & 0.06 \\
\hline TNM stage & 0.003 & & & 0.001 & & \\
\hline ApoAl & 0.02 & $0.587(0.352-0.979)$ & 0.04 & 0.01 & $0.607(0.413-0.893)$ & 0.01 \\
\hline ApoB & 0.36 & & & 0.83 & & \\
\hline ApoE & 0.18 & & & 0.39 & & \\
\hline
\end{tabular}

Note: Univariate analysis: Kaplan-Meier method; multivariate analysis: Cox proportional hazards regression model.

Abbreviations: BMI, body mass index; GLU, glucose; APLB, prealbumin; ALT, alanine aminotransferase; TB, total bilirubin; AFP, alpha-fetoprotein; ALB, prealbumin; GGT, glutamyl transpeptidase; HbAIC, hemoglobin AIC; CRP, C reactive protein; TC, total cholesterol; TG, triglycerides; HDL, high-density lipoprotein; LDL, low-density lipoprotein; OS, overall survival; DFS, disease free survival; HR, hazard ratio; $\mathrm{Cl}$, confidence interval. 


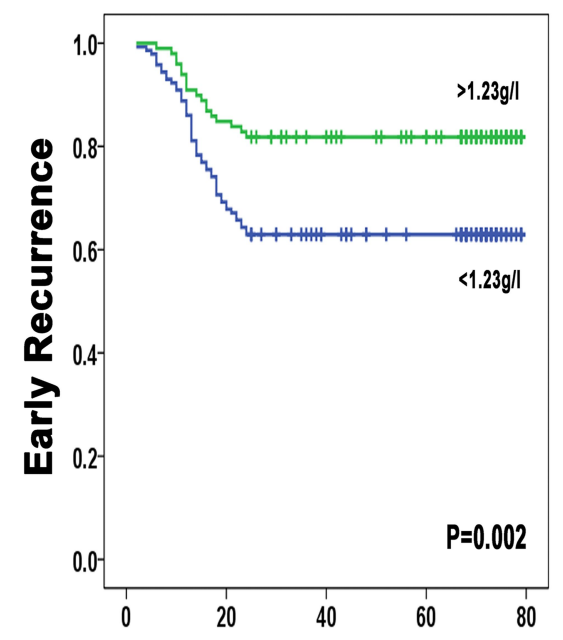

A Time(month)

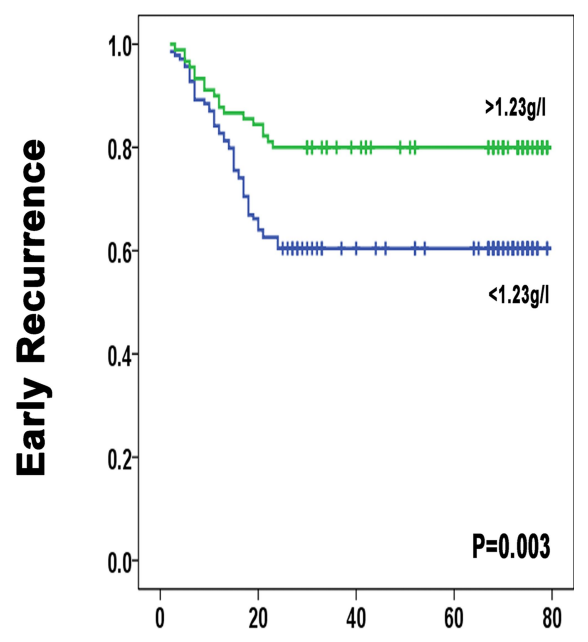

B Time(month)

Figure 3 Early recurrence curves in the training and validation cohorts according to ApoAl $\leq|.23 g /|$ or ApoA I >I.23g/l. (A) training cohort. (B) validation cohort.

cancer, although the underlying biological mechanisms are not fully understood.

The majority of HCC patients have an underlying chronic liver disease, therefore hepatic function is damaged obviously on these bases, which significantly influence lipid and apolipoprotein metabolism. Consequently, it has been suggested that plasma levels of lipids and apolipoproteins in HCC reflects on the hepatic cellular impairment status. We revealed that the majority of lipids and apolipoproteins were positively correlated with prealbumin and gamma-glutamyl transferase, two hepatic protein synthetic ability indices, and prompted that the pattern of changes in the lipid and apolipoprotein levels may be a good indicator of the hepatic protein synthetic ability and liver injury during the perioperative period after hepatectomy. A large number of studies have proved that liver injury parameters were closely related to poor prognosis in $\mathrm{HCC},{ }^{25,26}$ hence we hypothesize that circulating lipids and apolipoproteins could serve as indicators of prognosis after curative hepatectomy in routine clinical practice. In fact, the correlation of lipid and apolipoprotein levels in peripheral blood with the prognosis of HCC has been poorly investigated. ${ }^{27}$ Our study has revealed that although most of the lipids and apolipoproteins were not significantly associated with overall survival and tumor recurrence in both training and validation cohorts, a lower serum ApoA1 level was a negative prognostic factor of OS and DFS in patients with HCC in two independent cohorts. Therefore, serum ApoA1 might be a powerful and noninvasive biomarker to predict $\mathrm{HCC}$ prognosis for patients with curative surgery. Our results suggest that ApoA1 could also serve as an indicator of prognosis in patients with lower AFP concentration. So it may help surgeons to identify those surgical candidates at high risk of recurrence and poor prognosis before operation, and furthermore it would be a clinical monitoring indicator for patients with lower AFP concentration after operation since AFP is not feasible as a monitoring indicator, while ApoA1 is significantly correlated with the prognosis of patients of that sort.

Notably, the current study demonstrated no progressive relationship between ApoA1 expression and postoperative survival in patients with an AFP concentration of $\geq 400 \mu \mathrm{g} / \mathrm{L}$ that is generally associated with advanced stage and tumor progression, suggesting that ApoAl may be an efficient predictor of early prognosis status for patients. Various reports have suggested that patients with early recurrence generally have worse prognosis than those with late phase recurrence. ${ }^{28,29}$ Since the clinical outcome of patients who suffer from early recurrence is extremely dismal, ${ }^{30}$ identifying patients at high risk for early recurrence may help improve the prognosis of this population by rational adjuvant treatment. Although some research has shown the relationship between apolipoprotein levels and the survival of HCC patients, the correlation with HCC early recurrence has never been explored. ${ }^{31}$ Therefore, we next investigated prognostic value of ApoA1 to identify its value in HCC early recurrence and we further demonstrated here that low ApoA1 was an independent prognostic factor of poor early recurrence on multivariate analysis in two cohorts. As we 
Table 3 Univariate and Multivariate Analyses for Independent Variables Associated with HCC Early Recurrence

\begin{tabular}{|c|c|c|c|c|c|c|}
\hline \multirow[t]{3}{*}{ Variables } & \multicolumn{3}{|c|}{ Early Recurrence (Training Cohort) } & \multicolumn{3}{|c|}{ Early Recurrence (Validation Cohort) } \\
\hline & \multirow{2}{*}{$\begin{array}{l}\text { Univariate } \\
\mathbf{P}\end{array}$} & \multicolumn{2}{|l|}{ Multivariate } & \multirow{2}{*}{$\begin{array}{l}\text { Univariate } \\
\mathbf{P}\end{array}$} & \multicolumn{2}{|l|}{ Multivariate } \\
\hline & & HR (95\% Cl) & $\mathbf{P}$ & & HR (95\% CI) & $\mathbf{P}$ \\
\hline Gender & 0.45 & & & 0.005 & & \\
\hline Age(years) & 0.28 & & & 0.44 & & \\
\hline liver cirrhosis & 0.46 & & & 0.17 & & \\
\hline Hepatitis history & 0.45 & & & 0.18 & & \\
\hline BMI & 0.1 & & & 0.14 & & \\
\hline GLU & 0.78 & & & 0.11 & & \\
\hline APLB & 0.08 & & & 0.106 & & \\
\hline ALT & 0.87 & & & 0.01 & $1.747(1.026-2.975)$ & 0.04 \\
\hline TB & 0.87 & & & 0.41 & & \\
\hline AFP & 0.002 & & & 0.03 & & \\
\hline ALB & 0.67 & & & 0.7 & & \\
\hline GGT & 0.01 & & & $<0.001$ & $1.663(1.004-2.752)$ & 0.048 \\
\hline $\mathrm{CHOL}$ & 0.47 & & & 0.98 & & \\
\hline TG & 0.4 & & & 0.57 & & \\
\hline HDL & 0.86 & & & 0.88 & & \\
\hline LDL & 0.18 & & & 0.22 & & \\
\hline Tumor size & $<0.001$ & $2.869(I .76 I-4.673)$ & $<0.001$ & $<0.001$ & $2.084(1.275-3.407)$ & 0.003 \\
\hline Tumor number & 0.04 & & & 0.15 & & \\
\hline Tumor capsule & 0.01 & & & 0.44 & & \\
\hline Vascular invasion & $<0.001$ & $2.862(I .769-4.63 I)$ & $<0.001$ & $<0.001$ & $1.897(1.159-3.106)$ & 0.01 \\
\hline Edmondson grade & 0.003 & & & 0.15 & & \\
\hline TNM stage & $<0.001$ & & & 0.04 & & \\
\hline ApoAI & 0.002 & $0.5 \mathrm{I}(0.297-0.875)$ & 0.02 & 0.003 & $0.478(0.278-0.281)$ & 0.008 \\
\hline ApoB & 0.57 & & & 0.25 & & \\
\hline ApoE & 0.03 & $0.574(0.353-0.935)$ & 0.03 & 0.87 & & \\
\hline
\end{tabular}

Abbreviations: BMI, body mass index; GLU, glucose; APLB, prealbumin; ALT, alanine aminotransferase; TB, total bilirubin; AFP, alpha-fetoprotein; ALB, prealbumin; GGT, glutamyl transpeptidase; HbAIC, hemoglobin AIC; CRP, C reactive protein; TC, total cholesterol; TG, triglycerides; HDL, high-density lipoprotein; LDL, low-density lipoprotein; $\mathrm{HR}$, hazard ratio; $\mathrm{Cl}$, confidence interval.

know, HCCs larger than $5 \mathrm{~cm}$ always have a poor prognosis, ${ }^{32,33}$ and small HCCs less than $2 \mathrm{~cm}$ are always associated with a great prognosis, then we stratified patients into three subgroups according to tumor size. We observed a statistically significant difference in the curves for early HCC recurrence-free survival: patients with low ApoA1 had a higher recurrence rate in tumor size ranging from $2 \mathrm{~cm}$ to $5 \mathrm{~cm}$ subgroup. It is also worth noting that in AFP concentration of $<400 \mu \mathrm{g} / \mathrm{L}$ and TNM stage I subgroups, Kaplan-Meier survival curves showed that patients with lower ApoA1 levels had poor early recurrence rate in both cohorts. Consequently, we consider that ApoA1 might be utilized to identify a certain potentially manageable portion of HCC patients at high risk of early recurrence who are good candidates to receive more intensive surveillance and aggressive treatment post-surgery, especially for those with early stage.
To date, the precise mechanisms by which serum lipids and apolipoproteins contribute to the initiation and/or progression of cancer are not completely understood. Dyslipidaemia are known to be associated with the induction of oxidative stress as well as chronic inflammatory response and is closely related to insulin resistance, ${ }^{34-36}$ all of which have certain relationships with tumorigenesis. ${ }^{37,38}$ In vitro studies have confirmed that in several prostate, colon and breast cancer cells, high levels of cholesterol-rich lipid rafts in the plasma membrane in order to enhance cholesterol anabolism by reducing expression of ABCA1 which effuxes cholesterol in these cells, and meet the increasing requirements for cell proliferation. In addition, HDL and ApoA1 have attracted much interest by playing protective roles in cancer suggested from several large clinical studies. ${ }^{39,40}$ Furthermore, in mouse tumor 
models, ${ }^{41}$ ApoA1 not only prevented further development of the tumor but also led to tumor shrinkage. The anti-tumor effect of ApoA1 is profound and manifold, in large part by modulating the anti-tumor immune response. It promoted the accumulation of M1 antitumor phenotype macrophages, increased levels of tumor cell killing macrophages and recruited CD8T cells within the tumor microenvironment. But how ApoA1/HDL triggers these effects is not known and is currently under investigation. Notably, the current study demonstrated a progressive relationship between low serum ApoA1 levels and postoperative early recurrence and poor outcomes in HCC patients, illustrated that ApoA-1 might be a protective agent against liver cancer. Moreover, high density lipoprotein as a potential carrier for delivery of antitumoral drugs into hepatoma cells, ${ }^{42}$ and provision of human ApoA1 was therapeutic against established tumors, seems to supply a new treatment method in liver cancer.

There are some limitations to be considered in our study. Firstly, all participants enrolled with HCC experienced surgery. Since most patients who had accepted hepatic resection had well-compensated liver function, the serum lipids and apolipoproteins levels might not be significantly influenced by basic liver disease or HCC as compared to those with chronic liver diseases. This partly explained the phenomenon that no significant difference of lipids level was revealed between HCC and chronic liver diseases groups in our data. Secondly, the quantity of participants with chronic liver disease was relatively small compared to that of the HCC group, the power to detect a statistical difference might be weakened. Lastly, the present study was a retrospective single-centric study, further multicentric prospective studies are warranted to confirm our results.

In conclusion, the current study provided alterations in lipids and apolipoproteins patterns in patients with resectable HCC, chronic infection and benign liver disease. Among these factors, although ApoA1 was not suitable to be a diagnostic biomarker of HCC, it was confirmed as an independent prognosis factor for recurrence and survival in $\mathrm{HCC}$, and it might discriminate poor prognosis in patients with early-stage HCC. Further trials are necessary to clarify the mechanisms underlying anti-tumor effects of ApoA1.

\section{Acknowledgments}

This work was in part supported by National Key Sci-Tech Special Project of China (Grant 2012ZX10002010-001/ 002), the National Natural Science Foundation of China
(Grant 81302102), and the Basic Research Programs of Science and Technology Commission Foundation of Shanghai (Grants 13JC1401800, XBR2013074, and 13CG04)

\section{Author Contributions}

All authors made a significant contribution to the work reported, whether that is in the conception, study design, execution, acquisition of data, analysis and interpretation, or in all these areas; took part in drafting, revising or critically reviewing the article; gave final approval of the version to be published; and have agreed on the journal to which the article has been submitted; and agree to be accountable for all aspects of the work.

\section{Disclosure}

The authors declare that they have no conflict of interest.

\section{References}

1. Torre LA, Bray F, Siegel RL, et al. Global cancer statistics, 2012. $C A$ Cancer J Clin. 2015;65(2):87-108. doi:10.3322/caac.21262

2. Forner A, Llovet JM, Bruix J. Hepatocellular carcinoma. Lancet. 2012;379(9822):1245-1255. doi:10.1016/S0140-6736(11)61347-0

3. Portolani N, Coniglio A, Ghidoni S, et al. Early and late recurrence after liver resection for hepatocellular carcinoma: prognostic and therapeutic implications. Ann Surg. 2006;243(2):229-235. doi:10.1097/01.sla.0000197706.21803.a1

4. Chun JM, Kwon HJ, Sohn J, et al. after early recurrence in patients who underwent curative resection forhepatocellular carcinoma. J Surg Oncol. 2011;103(2):148-151. doi:10.1002/jso.21786

5. Tabuchi M, Kitayama J, Nagawa H. Hypertriglyceridemia is positively correlated with the development of colorectal tubular adenoma in Japanese men. World $J$ Gastroenterol. 2006;12(8):1261-1264. doi:10.3748/wjg.v12.i8.1261

6. Ulmer H, Borena W, Rapp K, et al. Serum triglyceride concentrations and cancer risk in a large cohort study in Austria. $\mathrm{Br} J$ Cancer. 2009;101(7):1202-1206. doi:10.1038/sj.bjc.6605264

7. Van Duijnhoven FJ, Bueno-De-Mesquita HB, Calligaro M, et al. Blood lipid and lipoprotein concentrations and colorectal cancer risk in the European Prospective Investigation into Cancer and Nutrition. Gut. 2011;60(8):1094-1102. doi:10.1136/gut.2010.225011

8. Shi J, Zhu L, Liu S, et al. A meta-analysis of case-control studies on the combined effect of hepatitis $\mathrm{B}$ and $\mathrm{C}$ virus infections in causing hepatocellular carcinoma in China. $B r \quad J$ Cancer. 2005;92 (3):607-612. doi:10.1038/sj.bjc.6602333

9. Fujii S, Koga S, Shono T, et al. Serum apoprotein A-I and A-II levels in liver diseases and cholestasis. Clin Chim Acta. 1981;115 (3):321-331. doi:10.1016/0009-8981(81)90245-X

10. Hachem H, Favre G, Raynal G, et al. Serum apolipoproteins A-I, A-II and $\mathrm{B}$ in hepatic metastases. Comparison with other liver diseases: hepatomas and cirrhosis. J Clin Chem Clin Biochem. 1986;24(3):161-166.

11. Motta M, Giugno I, Ruello P, et al. Lipoprotein (a) behaviour in patients with hepatocellular carcinoma. Minerva Med. 2001;92 (5):301-305.

12. Alsabti EA. Serum lipids in hepatoma. Oncology. 1979;36(1):11-14.

13. Ooi K, Shiraki K, Sakurai Y, et al. Clinical significance of abnormal lipoprotein patterns in liver diseases. Int J Mol Med. 2005;15 (4):655-660. 
14. Ahaneku JE, Taylor GO, Olubuyide IO, et al. Abnormal lipid and lipoprotein patterns in liver cirrhosis with and without hepatocellular carcinoma. J Pak Med Assoc. 1992;42(11):260-263.

15. Mustafa MG, Petersen JR, Ju H, et al. Biomarker discovery for early detection of hepatocellular carcinoma in hepatitis C-infected patients. Mol Cell Proteomics. 2013;12(12):3640-3652. doi:10.1074/mcp. M113.031252

16. Steel LF, Shumpert D, Trotter M, et al. A strategy for the comparative analysis of serum proteomes for the discovery of biomarkers for hepatocellular carcinoma. Proteomics. 2003;3(5):601-609. doi:10.1002/ pmic. 200300399

17. Sun HC, Zhang W, Qin LX, et al. Positive serum hepatitis B e antigen is associated with higher risk of early recurrence and poorersurvival in patients after curative resection of hepatitis B-related hepatocellular carcinoma. J Hepatol. 2007;47(5):684-690. doi:10.1016/j.jhep. 2007.06.019

18. Camp RL, Dolled-Filhart M, Rimm DL. X-tile: a new bio-informatics tool for biomarker assessment and outcome-based cut-point optimization. Clin Cancer Res. 2004;10(21):7252-7259. doi:10.11 58/1078-0432.CCR-04-0713

19. Yamamoto Y, Ikoma H, Morimura R, et al. Optimal duration of the early and late recurrence of hepatocellularcarcinoma after hepat-ectomy. World J Gastroenterol. 2015;21(4):1207-1215. doi:10.3748/wjg.v21.i4.1207

20. Cooper ME, Akdeniz A, Hardy KJ. Effects of liver transplantation and resection on lipid parameters: a longitudinal study. Aust $N Z$ Z J Surg. 1996;66(11):743-746. doi:10.1111/j.1445-2197.1996. tb00734.x

21. Marrero JA, Feng Z, Wang Y, et al. Alpha-fetoprotein, des-gamma carboxyprothrombin, and lectin-bound alpha-fetoprotein in earlyhepatocellular carcinoma. Gastroenterology. 2009;137(1):110-118. doi:10.1053/j.gastro.2009.04.005

22. Tremosini S, Forner A, Boix L, et al. Prospective validation ofan immunohistochemical panel (glypican 3, heat shock protein 70 andglutamine synthetase) in liver biopsies for diagnosis of very early hepatocellular carcinoma. Gut. 2012;61(10):1481-1487. doi:10.1136/gutjnl-2011-301862

23. Liu Y, Sogawa K, Sunaga M, et al. Increased concentrations of apo A-I and apo A-II fragments inthe serum of patients withhepatocellular carcinoma by magnetic beads-assisted MALDI-TOF mass spectrometry. Am J Clin Pathol. 2014;141(1):52-61. doi:10.1309/ AJCPBLFBNAP6N2UN

24. Cicognani C, Malavolti M, Morselli-Labate AM, et al. Serum lipid and lipoprotein patterns in patients with liver cirrhosis and chronic active hepatitis. Arch Intern Med. 1997;157(7):792-796. doi:10.1001/ archinte.1997.00440280120012

25. Faloppi L, Scartozzi M, Bianconi M, et al. The role of LDH serum levels in predicting global outcome in HCC patients treated with sorafenib: implicationsfor clinical management. BMC Cancer. 2014;14:110.

26. Scartozzi M, Faloppi L, Bianconi M, et al. The role of LDH serum levels in predicting global outcome in HCC patients undergoing TACE: implicationsfor clinical management. PLoS One. 2012;7(3): e32653. doi:10.1371/journal.pone.0032653

27. Yan X, Yao M, Wen X, et al. Elevated apolipoprotein B predicts poor postsurgery prognosis in patients with hepatocellularcarcinoma. Onco Targets Ther. 2019;12:1957-1964. doi:10.2147/OTT.S192631
28. Shimada M, Takenaka K, Gion T, et al. Prognosis of recurrent hepatocellular carcinoma: a10-year surgical experience in Japan Japan. Gastroenterology. 1996;111(3):720-726. doi:10.1053/ gast.1996.v111.pm8780578

29. Poon RT, Fan ST, Lo CM, et al. Intrahepatic recurrence after curative resection of hepatocellular carcinoma: long-term results oftreatment and prognostic factors. Ann Surg. 1999;229(2):216-222. doi:10.1097/ 00000658-199902000-00009

30. Poon RT, Fan ST, Ng IO, et al. Different risk factors and prognosis for early and late intrahepatic recurrence after resection ofhepatocellular carcinoma. Cancer. 2000;89(3):500-507. doi:10.1002/10970142(20000801)89:3<500::AID-CNCR4>3.0.CO;2-O

31. Mao M, Wang X, Sheng H, et al. A novel score based on serum apolipoprotein A-1 and C-reactive protein is a prognostic biomarker in hepatocellular carcinoma patients. BMC Cancer. 2018;18(1):1178. doi:10.1186/s12885-018-5028-8

32. Kim BK, Han KH, Park YN, et al. Prediction of microvascular invasion before curative resection of hepatocellular carcinoma. J Surg Oncol. 2008;97(3):246-252. doi:10.1002/jso.20953

33. Kaibori M, Ishizaki M, Matsui K, et al. Predictors of microvascular invasion before hepatectomy for hepatocellular carcinoma. J Surg Oncol. 2010;102(5):462-468. doi:10.1002/jso.21631

34. Ahotupa M, Suomela JP, Vuorimaa T, et al. Lipoprotein-specific transport of circulating lipid peroxides. Ann Med. 2010;42 (7):521-529. doi:10.3109/07853890.2010.510932

35. Moustafa T, Fickert P, Magnes C, et al. Alterations in lipid metabolism mediate inflammation, fibrosis, and proliferation in a mouse modelof chronic cholestatic liver injury. Gastroenterology. 2012;142 (1):140-151. doi:10.1053/j.gastro.2011.09.051

36. Kobayashi Y, Kashima H, Wu RC, et al. Mevalonate pathway antagonist suppresses formation of serous tubal intraepithelial carcinomaand ovarian carcinoma in mouse models. Clin Cancer Res. 2015;21 (20):4652-4662. doi:10.1158/1078-0432.CCR-14-3368

37. Freed-Pastor WA, Mizuno H, Zhao X, et al. Mutant p53 disrupts mammary tissue architecture via the mevalonate pathway. Cell. 2012;148(1-2):244-258. doi:10.1016/j.cell.2011.12.017

38. Noguti J, Andersen ML, Cirelli C, et al. Oxidative stress, cancer, and sleep deprivation: is there a logical link in this association? Sleep Breath. 2013;17(3):905-910. doi:10.1007/s11325-012-0797-9

39. Cao WM, Murao K, Imachi H, et al. A mutant high-density lipoprotein receptor inhibits proliferation of human breast cancer cells. Cancer Res. 2004;64(4):1515-1521. doi:10.1158/0008-5472.CAN03-0675

40. Mooberry LK, Nair M, Paranjape S, et al. Receptor mediated uptake of paclitaxel from a synthetic high density lipoprotein nanocarrier. J Drug Target. 2010;18(1):53-58. doi:10.3109/10611860903156419

41. Zamanian-Daryoush M, Lindner D, Tallant TC, et al. The cardioprotective protein apolipoprotein A1 promotes potent anti-tumorigenic effects. J Biol Chem. 2013;288(29):21237-21252. doi:10.1074/jbc. M113.468967

42. Lou B, Liao XL, Wu MP, et al. High-density lipoprotein as a potential carrier for delivery of a lipophilic antitumoral drug intohepatoma cells. World J Gastroenterol. 2005;11(7):954-959. doi:10.3748/wjg.v11.i7.954 


\section{Publish your work in this journal}

OncoTargets and Therapy is an international, peer-reviewed, open access journal focusing on the pathological basis of all cancers, potential targets for therapy and treatment protocols employed to improve the management of cancer patients. The journal also focuses on the impact of management programs and new therapeutic

Submit your manuscript here: https://www.dovepress.com/oncotargets-and-therapy-journal agents and protocols on patient perspectives such as quality of life, adherence and satisfaction. The manuscript management system is completely online and includes a very quick and fair peer-review system, which is all easy to use. Visit http://www.dovepress.com/ testimonials.php to read real quotes from published authors. 